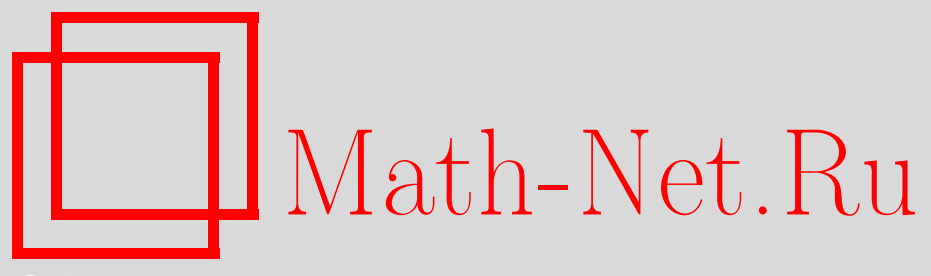

А. М. Ильин, Б. И. Сулейманов, Асимптотика специального решения уравнения Абеля, связанного с особенностью сборки, Матем. сб., 2006, том 197, номер 1, 55-70

DOI: https://doi.org/10.4213/sm1119

Использование Общероссийского математического портала Math-Net.Ru подразумевает, что вы прочитали и согласны с пользовательским соглашением http://www . mathnet.ru/rus/agreement

Параметры загрузки:

IP : 3.85 .73 .92

26 апреля 2023 г., 05:24:19

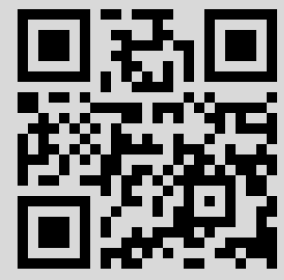




\author{
А. М. Ильин, Б. И. Сулейманов
}

\title{
Асимптотика специального решения уравнения Абеля, связанного с особенностью сборки
}

Рассмотрено специальное решение обыкновенного дифференциального уравнения Абеля первого рода $u_{x}^{\prime}+u^{3}-t u-x=0$, которое описывает поведение широкого ряда решений дифференциальных уравнений с частными производными с малым параметром около точек сборки их медленно меняющихся положений равновесия. Доказано существование этого специального решения. Построена и обоснована его асимптотика при $|x| \rightarrow \infty, t \rightarrow-\infty$.

Библиография: 4 названия.

\section{Введение}

В работе исследовано специальное решение обыкновенного дифференциального уравнения (ОДУ)

$$
u_{x}^{\prime}+u^{3}-t u-x=0
$$

Это решение ОДУ $(0.1)$, определенное на всей плоскости $(x, t)$, связано с решениями широкого ряда уравнений математической физики с малым параметром. В работе [1] показано, что оно является главным членом внутренних асимптотических разложений в одном из вариантов, когда внешние разложения решений имеют особенности типа сборки [2]. В другом варианте [1], [3] главным членом внутренних асимптотических разложений является решение похожего уравнения второго порядка.

Главным содержанием работы является исследование равномерной и полной асимптотики данного специального решения ОДУ (0.1) при $|x| \rightarrow \infty, t \rightarrow-\infty$ (существование решения и его асимптотика при фиксированном значении $t$ были получены ранее [1]). Кроме того, доказана гладкость построенного решения и получены равномерные асимптотики всех его производных.

\section{§ 1. Вспомогательные леммы}

В этом параграфе все функции для простоты считаются достаточно гладкими.

Работа выполнена при поддержке Российского фонда фундаментальных исследований (гранты №№ 02-01-00768, 04-02-97503, 06-01-00124) и Программы поддержки ведущих научных школ РФ (грант № НШ-1446.2003.1). 
ЛЕмма 1.1. Пусть функиия $y(x)$ есть решение ОДУ

$$
y^{\prime}(x)=g(x, y)
$$

а функция $z(x)$, совпадая с $y(x)$ при $x=x_{0}$ на содержащем эту точку промежутке, удовлетворяет неравенству

$$
z^{\prime}(x)<g(x, z(x))
$$

Тогда на правой части этого промежутка (при $\left.x>x_{0}\right)$ справедливо неравенство

$$
z(x)<y(x)
$$

а на его левой части (при $\left.x<x_{0}\right)$ - неравенство

$$
z(x)>y(x)
$$

Знаки неравенств (1.2), (1.3) меняются на обратные в случае противоположного знака в неравенстве (1.1).

Доказательство легко провести методом от противного (см. также работу [4; гл. 1])

Лемма 1.2. Пусть на луче $L: x \leqslant x_{1}<\infty$, для некоторого $\beta>0$ и функиии $Q(x)>1$ функиия $v(x)$ удовлетворяет неравенству

$$
v^{\prime}(x)+\beta Q(x) v(x)<0,
$$

u пусть при $x \rightarrow-\infty$

$$
v(x)=o(\exp (-\beta x))
$$

Тогда при $x<x_{1}$

$$
v(x)<0 .
$$

ДокАзАТЕЛЬСтво. Допустим противное: либо функция $v(x)$ положительна всюду при $x \leqslant x_{1}$, либо $v\left(x_{2}\right)=0$ для некоторого $x_{2} \leqslant x_{1}$. Во втором случае согласно лемме 1.1 функция $v(x)$ положительна при $x<x_{2}$. Поэтому в обоих случаях существует точка $x_{3} \leqslant x_{1}$ такая, что функция $v(x)$ положительна при $x \leqslant x_{3}$.

На таком луче были бы справедливы неравенство

$$
v^{\prime}(x)+\beta v(x)<v^{\prime}(x)+\beta Q(x) v(x)<0
$$

и, стало быть, неравенство

$$
(v(x) \exp (\beta x))_{x}^{\prime}<0
$$

После интегрирования последнего неравенства на промежутке $\left[x, x_{3}\right]$ в этом случае получалось бы неравенство

$$
v(x) \geqslant v\left(x_{3}\right) \exp \left(\beta\left(x_{3}-x\right)\right),
$$

противоречащее условию (1.5). Лемма 1.2 доказана. 
ЛЕмма 1.3. Пусть на интервале $I:-\infty<x_{1} \leqslant x<x_{2} \leqslant \infty$, функция $v(x)$ для $\beta>0$ удовлетворяет неравенству (1.4) с функиией $Q(x)>1$. Тогда при $x \geqslant x_{1}$ справедлива оценка

$$
v(x) \leqslant\left|v\left(x_{1}\right)\right| \exp \left(\beta\left(x_{1}-x\right)\right) .
$$

ДокАЗАтЕльство. При отрицательных значениях $v(x)$ неравенство (1.7) выполнено. Пусть $u\left(x_{3}\right)>0$ для некоторого $x_{3}>x_{1}$. Тогда из неравенства (1.4) следует, что $u(x)>0$ для всех $x$ при $x_{1} \leqslant x \leqslant x_{3}$. Поэтому на отрезке $\left[x_{1}, x_{3}\right]$ справедливо неравенство (1.6) Интегрируя это неравенство от $x_{1}$ до $x_{3}$, приходим к оценке $(1.7)$ для значения $u\left(x_{3}\right)$. Лемма 1.3 доказана.

\section{§ 2. Асимптотика решения $u(x, t)$ при $|x| \rightarrow \infty$ и ограниченных $t$}

Прежде всего определим исследуемое решение уравнения (0.1). С этой целью рассмотрим следующую функцию $H(x, t)$ :

1) при $t \leqslant 0 \quad H(x, t)$ - единственное решение кубического уравнения

$$
H^{3}-t H-x=0
$$

2) в области

$$
t>0, \quad x<x_{c}(t)=\frac{2}{\sqrt{27}} t^{3 / 2}
$$

$H(x, t)$ - непрерывное отрицательное решение уравнения (2.1);

3) наконец, при

$$
t>0, \quad x \geqslant x_{c}(t)
$$

$H(x, t)$ - непрерывное положительное решение уравнения (2.1).

В этом параграфе для любого фиксированного значения $t$ строится решение ОДУ $(0.1)$ и изучаются его асимптотические разложения при $x \rightarrow \infty$ и при $x \rightarrow-\infty$. Эти асимптотические разложения равномерны относительно $t$ при $|t| \leqslant T$, где $T$ - любое фиксированное положительное число (равномерность асимптотик нетрудно заметить из приводимых ниже построений и доказательств). Поэтому в дальнейшем для избежания громоздкости зависимость от параметра $t$ в обозначениях довольно часто опускается.

Пусть $x_{0}$ - какое-нибудь отрицательное число. Посредством $u_{a}(x, t)$ обозначим решение ОДУ $(0.1)$ такое, что $u_{a}\left(x_{0}, t\right)=a<H\left(x_{0}, t\right)$, и покажем, что при некотором $a$ это и есть искомое решение. Через $\mathbb{M}$ обозначим множество таких $a<H\left(x_{0}, t\right)$, что графики функций $u_{a}(x, t)$ и $H(x, t)$ имеют какую-нибудь общую точку при $x<x_{0}$. Так как $\frac{\partial H}{\partial x}\left(x_{0}, t\right)>0$, a $\frac{\partial u_{a}}{\partial x}\left(x_{0}, t\right)=-a^{3}+t a+x_{0}$ мало при а достаточно близких к $H\left(x_{0}, t\right)$, то отсюда и из непрерывной зависимости решения $u_{a}(x, t)$ от параметра $a$ вытекает, что множество $\mathbb{M}$ непусто (ему принадлежат все $a<H\left(x_{0}, t\right)$, достаточно близкие к $\left.H\left(x_{0}, t\right)\right)$ и открыто. 
Кроме того, множество $\mathbb{M}$ ограничено снизу. Действительно, если $a$ - достаточно большое отрицательное число, то

$$
\frac{\partial u_{a}}{\partial x}\left(x_{0}, t\right)=-a^{3}+t a+x_{0}>\frac{\partial H}{\partial x}\left(x_{0}, t\right)>0
$$

и

$$
\frac{\partial^{2} u_{a}}{\partial x^{2}}(x, t)=\left(-3 u_{a}^{2}(x, t)+t\right) \frac{\partial u_{a}}{\partial x}(x, t)+1
$$

меньше нуля при $x=x_{0}$.

Покажем, что для такого $a$ неравенство $\frac{\partial^{2} u_{a}}{\partial x^{2}}(x, t)<0$ справедливо и при всех $x<x_{0}$. Допустим противное. Пусть точка $x_{1}-$ ближайшая слева к точке $x_{0}$, в которой функция $\frac{\partial^{2} u_{a}}{\partial x^{2}}(x, t)$ равна нулю. Тогда на интервале $\left(x_{1}, x_{0}\right]$ при уменьшении $x$ возрастают производная $\frac{\partial u_{a}}{\partial x}(x, t)$ и, как следствие, функция $u_{a}^{2}(x, t)$. Поэтому в точке $x_{1}$ правая часть равенства $(2.2)$ меньше нуля, что противоречит сделанному предположению.

Следовательно, при $x<x_{0}$ функция $u_{a}(x, t)$ меньше линейной функции $a+$ $\frac{\partial H}{\partial x}\left(x_{0}, t\right)\left(x-x_{0}\right)$. И так как функция $H(x, t)$ выпукла вниз, то при $x<x_{0}$ графики функций $u_{a}(x, t)$ и $H(x, t)$ не пересекаются.

Искомую функцию $u(x, t)$ построим при $x \leqslant x_{0}$ как решение $u_{\alpha}(x, t)$, где

$$
\alpha=\inf (\mathbb{M})
$$

и продолжим его при $x \geqslant x_{0}$ как решение ОДУ $(0.1)$.

Теорема 2.1. При всех $t$ решение $u(x, t)$ есть гладкая монотонно возрастающая функиия переменной $x$ такая, что $u(x, t)<H(x, t)$; это есть единственное решение ОДУ (0.1), удовлетворяющее условию

$$
u(x, t)-H(x, t) \rightarrow 0
$$

при $x \rightarrow-\infty$.

ДоКАЗАТЕЛЬСТвО. Из непрерывной зависимости решений ОДУ (0.1) от их значений в точке $x_{0}$ вытекает, что решение $u(x, t)$ не может стремиться к $-\infty$ при конечном значении $x \leqslant x_{0}$ (в этом легко убедиться, повторив рассуждения, примененные выше при доказательстве ограниченности снизу множества $\mathbb{M}$ ).

Следовательно, гладкая функция $u(x, t)$ определена для всех значений $x \leqslant x_{0}$ и для этих значений $x$ справедливо неравенство $u(x, t)<H(x, t)$. Соотношение (2.3) легко доказывается от противного. Если оно не выполнено, то для достаточно большого отрицательного $x_{1}$ справедливы неравенства

$$
\begin{gathered}
u\left(x_{1}, t\right)-H\left(x_{1}, t\right)<-\delta<0, \\
H^{2}\left(x_{1}, t\right)>2 t+\frac{2}{\delta} .
\end{gathered}
$$


Тогда

$$
\begin{aligned}
& \frac{\partial u}{\partial x}\left(x_{1}, t\right)=-u\left(x_{1}, t\right)^{3}+t u\left(x_{1}, t\right)+x_{1}+\left(H^{3}(x, t)-t H\left(x_{1}, t\right)-x_{1}\right) \\
& \quad=-\left(u\left(x_{1}, t\right)-H\left(x_{1}, t\right)\right)\left(u^{2}\left(x_{1}, t\right)+u\left(x_{1}, t\right) H\left(x_{1}, t\right)+H^{2}\left(x_{1}, t\right)-t\right) \\
& \quad \geqslant-\left(u\left(x_{1}, t\right)-H\left(x_{1}, t\right)\right)\left(\frac{H^{2}\left(x_{1}, t\right)}{2}-t\right)>1
\end{aligned}
$$

И

$$
\begin{aligned}
\frac{\partial^{2} u}{\partial x^{2}}\left(x_{1}, t\right) & =\frac{\partial u}{\partial x}\left(x_{1}, t\right)\left(t-3 u^{2}\left(x_{1}, t\right)\right)+1 \\
& <\frac{\partial u}{\partial x}\left(x_{1}, t\right)\left(t-3 H^{2}\left(x_{1}, t\right)\right)+1<0 .
\end{aligned}
$$

Теперь снова, почти дословно повторяя рассуждения, проведенные при доказательстве ограниченности снизу множества $\mathbb{M}$, из двух последних неравенств получаем, что при $x<x_{1}$ функция $u(x, t)$ меньше линейной функции $u\left(x_{1}, t\right)+\left(x-x_{1}\right)$ (противоречие с определением $u(x, t)=u_{\alpha}(x, t)$, где $\alpha=\inf (\mathbb{M}))$. Из соотношения (2.3) вытекает, в частности, что

$$
\lim _{x \rightarrow-\infty} \frac{u(x, t)}{x^{1 / 3}}=1 .
$$

Допустим, что при достаточно больших значениях $-x$ существует отличное от $u(x, t)$ решение ОДУ $(0.1) u_{1}(x, t)$, которое также удовлетворяет предельному условию (2.6). Тогда функция $r(x, t)=\left|u(x, t)-u_{1}(x, t)\right|$ при $x \rightarrow-\infty$ удовлетворяет соотношению

$$
(\ln r(x, t))_{x}^{\prime}=t-u_{1}^{2}(x, t)-u(x, t) u_{1}(x, t)-u^{2}(x, t)=-3 x^{2 / 3}+o\left(x^{2 / 3}\right),
$$

влекущему за собой вывод об экспоненциальном росте $r(x, t)$ при $x \rightarrow-\infty$. Это противоречит предельным соотношениям (2.6) для решений $u(x, t)$ и $u_{1}(x, t)$.

Далее заметим, что точка экстремума любого решения ОДУ (0.1) - та и лишь та, в которой данное решение совпадает с $H(x, t)$, и что такая точка - это точка минимума. Очевидно, что функция $u(x, t)$ монотонна при $x \leqslant x_{0}$. Поэтому из монотонности функции $H(x, t)$ при всех $x$ следует, что и при $x>x_{0}$ имеет место неравенство $u(x, t)<H(x, t)$. Значит, справедливо утверждение теоремы.

Теорема 2.2. Решение $и(x, t)$ является непрерьвной функцией обеих переменных.

ДокАзАтельство. Очевидно, что достаточно доказать непрерывность функции $u\left(x_{0}, t\right)$ по переменной $t$. Допустим противное - функция $u\left(x_{0}, t\right)$ разрывна в точке $t_{0}$. Это означает существование такой последовательности $t_{n} \rightarrow t_{0}$, что

$$
\left|u\left(x_{0}, t_{n}\right)-u\left(x_{0}, t_{0}\right)\right|>\gamma>0 .
$$

В случае

$$
u\left(x_{0}, t_{n}\right)-u\left(x_{0}, t_{0}\right)>\gamma>0
$$


рассмотрим решение $u_{b}(x, t)$ уравнения $(0.1)$, которое при $x=x_{0}$ равно $b=$ $u\left(x_{0}, t_{0}\right)+\gamma / 2$. Согласно определению множества $\mathbb{M}$ график функции $u_{b}\left(x, t_{0}\right)$ пересекается с графиком функции $H\left(x, t_{0}\right)$ при каком-нибудь $x<x_{0}$. В силу непрерывности $H(x, t)$ и $u_{b}(x, t)$ их графики пересекаются при $t=t_{n}$, близком к $t_{0}$ в точке, расположенной левее точки $x_{0}$. Это противоречит неравенству $u\left(x_{0}, t_{n}\right)-u_{b}\left(x_{0}, t_{n}\right)>\gamma / 2$ и определению множества $\mathbb{M}$.

Пусть теперь $u\left(x_{0}, t_{n}\right)-u\left(x_{0}, t_{0}\right)<-\gamma<0$. Тогда рассмотрим решение $u_{c}(x, t)$ уравнения $(0.1)$, которое при $x=x_{0}$ равно $c=u\left(x_{0}, t_{0}\right)-\gamma / 2$. Для этого решения найдется точка $x_{1}<x_{0}$ такая, что при $t=t_{0}$ в ней выполнены неравенства $(2.4),(2.5)$. Следовательно, в точке $x_{1}$ эти неравенства справедливы для $u_{c}(x, t)$ и при $t=t_{n}$, близких к $t_{0}$. Из рассуждений, приведенных при доказательстве теоремы 2.1, следует, что в этом случае решение $u_{c}\left(x, t_{n}\right) \rightarrow-\infty$ при $x \rightarrow-\infty$ не медленнее линейной функции. Итак, при $t=t_{n}$ мы имеем два решения уравнения (0.1): построенное в теореме 2.1 решение $u\left(x, t_{n}\right)$, для которого справедливо соотношение $(2.3)$, и решение $u_{c}\left(x, t_{n}\right)$. Хотя $u_{c}\left(x_{0}, t_{n}\right)-u\left(x_{0}, t_{n}\right)>\gamma / 2>0$, но при достаточно больших отрицательных $x$ справедливо обратное неравенство: $u_{c}\left(x, t_{n}\right)<u\left(x, t_{n}\right)$. Следовательно, их графики пересекаются, что противоречит единственности решения начальной задачи для уравнения (0.1). Теорема доказана.

Теорема 2.3. Для любого $t$ бункиия $u(x, t)$ при $x \rightarrow-\infty$ и при $x \rightarrow \infty$ разлагается в асимптотический ряд

$$
u(x, t)=x^{1 / 3}\left(1+\sum_{j=2}^{\infty} c_{j}(t) x^{-j / 3}\right)
$$

где коэфбициенты $c_{j}(t)$ - это многочлены, которые однозначно определяются после формальной подстановки ряда (2.7) в уравнение (0.1). Для любого положительного T асимптотическое разложение (2.7) равномерно при $|t| \leqslant T$, а именно для любого натурального $n$ существуют постоянные $M_{n}(T)$ такие, что при $|t| \leqslant T,|x|>1$ справедливы неравенства

$$
\left|u(x, t)-x^{1 / 3}\left(1+\sum_{j=2}^{2 n} c_{j}(t) x^{-j / 3}\right)\right| \leqslant M_{n}(T)|x|^{-2 n / 3} .
$$

ДокАЗАТЕЛЬСтво. Нетрудно видеть, что коэффициенты $c_{j}(t)$ - это многочлены $\left(c_{2}(t)=t / 3, c_{3}(t)=c_{4}(t)=0, \ldots\right)$. Рассмотрим функции

$$
\begin{aligned}
& Y_{n}^{-}(x, t)=x^{1 / 3}\left(1+\sum_{j=2}^{2 n} c_{j}(t) x^{-j / 3}\right)-M_{n}(T) x^{-2 n / 3}, \\
& Y_{n}^{+}(x, t)=x^{1 / 3}\left(1+\sum_{j=2}^{2 n} c_{j}(t) x^{-j / 3}\right)+M_{n}(T) x^{-2 n / 3} .
\end{aligned}
$$


Покажем, что для любого натурального $n>2$ существуют такие положительные постоянные $M_{n}(T), B_{n}(T)$, что в каждой из полуполос

$$
\begin{aligned}
\mathrm{E}_{l} & =\left(x, t: x<-B_{n}(T),|t| \leqslant T\right), \\
\mathrm{E}_{r} & =\left(x, t: x>B_{n}(T),|t| \leqslant T\right)
\end{aligned}
$$

функции $Y_{n}^{-}(x, t)$ и $Y_{n}^{+}(x, t)$ удовлетворяют неравенствам

$$
\begin{aligned}
& \left(Y_{n}^{-}(x, t)\right)_{x}^{\prime}+\left(Y_{n}^{-}(x, t)\right)^{3}-t Y_{n}^{-}(x, t)-x<0, \\
& \left(Y_{n}^{+}(x, t)\right)_{x}^{\prime}+\left(Y_{n}^{+}(x, t)\right)^{3}-t Y_{n}^{+}(x, t)-x>0 .
\end{aligned}
$$

Обозначим

$$
Z_{n}(x, t)=x^{1 / 3}\left(1+\sum_{j=2}^{2 n} c_{j}(t) x^{-j / 3}\right)
$$

Тогда

$$
\begin{aligned}
& \left(Y_{n}^{-}(x, t)\right)_{x}^{\prime}+\left(Y_{n}^{-}(x, t)\right)^{3}-t Y_{n}^{-}(x, t)-x \\
& =\left(Z_{n}(x, t)\right)_{x}^{\prime}+\frac{2 n}{3} M_{n}(T) x^{-(2 n+3) / 3}+Z_{n}^{3}(x, t) \\
& \quad-3 Z_{n}^{2}(x, t) M_{n}(T) x^{-2 n / 3}+3 Z_{n}(x, t) M_{n}^{2}(T) x^{-4 n / 3} \\
& \quad-M_{n}^{3}(T) x^{-2 n}-t Z_{n}(x, t)+t M_{n}(T) x^{-2 n / 3}-x .
\end{aligned}
$$

Так как при формальной подстановке ряда (2.7) в ОДУ (0.1) коэффициенты при всех степенях $x$ обращаются в нуль, то при подстановке $Z_{n}(x, t)$ в это уравнение обратятся в нуль коэффициенты при тех степенях $x$, показатели которых больше, чем $(2-2 n) / 3$. Следовательно,

$$
\left(Z_{n}(x, t)\right)_{x}^{\prime}+Z_{n}^{3}(x, t)-t Z_{n}(x, t)-x=x^{(2-2 n) / 3} K_{n}\left(t, x^{-1 / 3}\right),
$$

где $K_{n}\left(t, x^{-1 / 3}\right)$ - это многочлен относительно обоих аргументов.

Ясно, что правая часть равенства (2.13) есть сумма

$$
x^{(2-2 n) / 3} \sum_{j=0}^{m_{n}} S_{j}(t) x^{-j / 3},
$$

главный член которой $S_{0}(t)=-3 M_{n}(T)+K_{n}(t, 0)$ отрицателен при $|t|<T$ и при достаточно большом значении $M_{n}(T)$. Следовательно, для достаточно больших значений $B_{n}(T)$ в полуполосах $(2.9)$ и (2.10) действительно справедливо неравенство (2.11). Аналогичным образом доказывается справедливость в этих полуполосах и неравенства $(2.12)$.

Из неравенств (2.11) и (2.12) следует, что для достаточно больших значений $B_{n}(T)$ разности

$$
R_{-}(x, t)=Y_{n}^{-}(x, t)-u(x, t), \quad R_{+}(x, t)=u(x, t)-Y_{n}^{+}(x, t)
$$

в полуполосах (2.9), (2.10) удовлетворяют неравенствам

$$
\left.R_{\mp}(x, t)_{x}^{\prime}+Q_{\mp}(x, t) R_{\mp}(x, t)\right)<0
$$


с коэффициентами

$$
Q_{\mp}(x, t)=u^{2}(x, t)+u(x, t) Y_{n}^{\mp}(x, t)+\left(Y_{n}^{\mp}(x, t)\right)^{2}-t>\frac{\left(Y_{n}^{\mp}(x, t)\right)^{2}}{2}-t>1 .
$$

Согласно лемме 1.2 из последних неравенств и соотношения (2.3) вытекает, что в полуполосе (2.9) оценки (2.8) имеют место. Аналогичным образом согласно лемме 1.3 из неравенств (2.14), (2.15) вытекает, что в полуполосе (2.10) справедливы оценки (2.8). Действительно, из леммы 1.3 следует, что если функции $R_{ \pm}(x, t)$ положительны, то они при этом экспоненциально малы при $x \rightarrow \infty$. С учетом непрерывности $u(x, t)$ отсюда следует, что оценки (2.8) для достаточно больших значений постоянных $M_{n}(T)$ будут справедливы и при всех $|x|>1$, $|t| \leqslant T$. Теорема доказана.

ТеОрема 2.4. Функиия $и(x, t)$ бесконечно дифберенцируема для всех $x$ и $t$.

ДокАЗАТЕЛЬство. Поскольку в теореме 2.2 непрерывность функции $u(x, t)$ доказана, то непосредственно из уравнения (0.1) вытекает непрерывность производной $\frac{\partial u(x, t)}{\partial x}$, а последовательное дифференцирование обеих частей этого уравнения приводит к заключению о существовании и непрерывности всех производных $\frac{\partial^{m} u(x, t)}{\partial x^{m}}$. цию

Для доказательства существования производной $\frac{\partial u(x, t)}{\partial t}$ рассмотрим функ-

$$
w(x, t, h)=\frac{u(x, t+h)-u(x, t)}{h} .
$$

Из уравнения (0.1) следует, что

$$
\frac{\partial w(x, t, h)}{\partial x}=w(x, t, h)\left(t-u^{2}(x, t+h)-u(x, t+h) u(x, t)-u^{2}(x, t)\right)+u(x, t+h) .
$$

Если обозначить

$$
g(x, t, h)=\int_{0}^{x}\left(u^{2}(\xi, t+h)+u(\xi, t+h) u(\xi, t)+u^{2}(\xi, t)-t\right) d \xi
$$

и учесть, что в силу справедливости соотношения $(2.3) w(x, t, h) \rightarrow 0$ при $x \rightarrow$ $-\infty$, то из соотношения (2.16) следует формула

$$
w(x, t, h)=\exp (-g(x, t, h)) \int_{-\infty}^{x} \exp (g(\xi, t, h)) u(\xi, t+h) d \xi
$$

Равномерная сходимость последнего из выписанных интегралов обеспечена неравенством (2.8). И так как $u(x, t)$ - непрерывная функция, обладающая асимптотикой (2.7), то из формулы (2.17) следует существование функции

$$
w(x, t)=\lim _{h \rightarrow 0} w(x, t, h)=\frac{\partial u}{\partial t}(x, t)=\exp (-g(x, t)) \int_{-\infty}^{x} \exp (g(\xi, t)) u(\xi, t) d \xi,
$$


где $g(x, t)=\int_{0}^{x}\left(3 u^{2}(\xi, t)-t\right) d \xi$. Из этой формулы вытекает непрерывность $\frac{\partial u}{\partial t}(x, t)$ на всей плоскости $(x, t)$. Дифференцируя обе части уравнения $(0.1)$ по $t$, получаем уравнение

$$
\frac{\partial w(x, t)}{\partial x}=\left(t-3 u^{2}(x, t)\right) w(x, t)+u(x, t) .
$$

Из этого уравнения вытекают существование и непрерывность всех производных

$$
\frac{\partial^{m} w}{\partial x^{m}}(x, t)=\frac{\partial^{m+1} u}{\partial t \partial x^{m}}(x, t) .
$$

Применяя к уравнению (2.19) тот же прием, что и к уравнению $(0.1)$, получаем существование и непрерывность

$$
\frac{\partial w}{\partial t}(x, t)=\frac{\partial^{2} u}{\partial t^{2}}(x, t)
$$

Далее, из уравнения для $\frac{\partial^{2} u}{\partial t^{2}}(x, t)$ следуют существование и непрерывность всех производных $\frac{\partial^{m+2} u}{\partial t^{2} \partial x^{m}}(x, t)$. Существование и непрерывность всех остальных производных получаются по индукции. Теорема доказана.

Теорема 2.5. Асимптотическое разложение допускает почленное дифференцирование любого порядка по переменным $x$ u $t$. Для любого положительного $T$ и произвольных натуральных чисел $k, m, n$ существуют такие постоянные $M_{k, m, n}(T)$, что при $|t| \leqslant T,|x|>1$ справедливы неравенства

$$
\left|\frac{\partial^{k+m}}{\partial x^{k} \partial t^{m}}\left(u(x, t)-x^{1 / 3}\left(1+\sum_{j=2}^{2 n} c_{j}(t) x^{-j / 3}\right)\right)\right| \leqslant M_{k, m, n}(T)|x|^{-k-2 n / 3} .
$$

ДокАЗАТЕльство. Так как для функции $u(x, t)$ справедливо асимптотическое разложение $(2.7)$, то из уравнения (0.1) вытекает, что производная $\frac{\partial u}{\partial x}(x, t)$ также разлагается в асимптотический ряд по степеням $x$ при $|x| \rightarrow \infty$. И поскольку степенные асимптотические ряды можно почленно интегрировать, то отсюда следует, что ряд (2.7) можно почленно дифференцировать по $x$. Далее, так же показывается, что возможно почленное дифференцирование ряда (2.7) по $x$ любое число раз.

Докажем справедливость утверждения теоремы для функции $\frac{\partial u}{\partial t}(x, t)$. Из соотношения (2.18) следует ее представление в виде

$$
\begin{aligned}
& \frac{\partial u}{\partial t}(x, t)=w(x, t) \\
& \quad=\exp (-g(x, t))\left(\int_{-\infty}^{2 x} \exp (g(\xi, t)) u(\xi, t) d \xi+\int_{2 x}^{x} \exp (g(\xi, t)) u(\xi, t) d \xi\right),
\end{aligned}
$$


где функция $g(x, t)$ при $x \rightarrow-\infty$ имеет асимптотику

$$
g(x, t)=\frac{9}{5} x^{5 / 3}+t x+g_{2}(t) x^{2 / 3}+g_{1}(t) x^{1 / 3}+g_{0}(t)+d \ln x+\sum_{j=0}^{\infty} g_{-j}(t) x^{-2 j / 3} .
$$

Данное представление (2.21) и оценки (2.8) позволяют легко сделать вывод о том, что при $x \rightarrow-\infty$ модуль функции $w(x, t)$ растет не быстрее некоторой степени $|x|$.

С другой стороны, если считать решение $u(x, t)$ известным, то соотношение (2.19) можно рассматривать как линейное ОДУ на функцию $w(x, t)$. После подстановки в ОДУ $(2.19)$ вместо $u(x, t)$ его асимптотических разложений $(2.7)$, нетрудно убедиться в том, что данное ОДУ при $x \rightarrow \pm \infty$ обладает единственными формальными решениями вида

$$
w_{f}(x, t)=\frac{1}{3 x^{1 / 3}}+\sum_{j=2}^{\infty} w_{j}(t) x^{-j / 3},
$$

где $w_{j}(t)$ суть вполне конкретные полиномы. Рассмотрим функции

$$
W_{n}^{ \pm}(x, t)=\frac{1}{3 x^{1 / 3}}+\sum_{j=2}^{n-1} \frac{w_{j}(t)}{x^{j / 3}}+M_{0,1, n}(T) x^{-2 n / 3}
$$

зависящие от достаточно больших постоянных $M_{0,1, n}(T)$. Применим к этим функциям и к функции $w(x, t)$ рассуждения, аналогичные тем, которые ранее использовались при доказательстве теоремы 2.2 по отношению к функциям $Y_{n}^{ \pm}(x, t)$ и $u(x, t)$. Тем самым, асимптотическое разложение доказано для производной $\frac{\partial u}{\partial t}(x, t)$. Так как асимптотические ряды можно почленно интегрировать, то утверждение теоремы справедливо для первой производной.

Окончание доказательства получается по индукции с учетом линейных ОДУ для функций $\frac{\partial^{k+m}}{\partial x^{k} \partial t^{m}} u(x, t)$ (аналогов линейного ОДУ $(2.19)$ для производной $\left.\frac{\partial u}{\partial t}(x, t)\right)$. Теорема доказана.

\section{§ 3. Асимптотика решения при $t \rightarrow-\infty$}

Введем переменные

$$
s=x|t|^{-3 / 2}, \quad v=u|t|^{-1 / 2} .
$$

В этих переменных при $t \neq 0$ ОДУ $(0.1)$ примет вид

$$
|t|^{-5 / 2} v_{s}=s-v-v^{3} .
$$

В дальнейшем под $v(s, t)$ будем понимать именно то решение данного ОДУ, которое с функцией $u(x, t)$ связано заменами (3.1). 
Согласно теореме 2.1 при $t<0$ функция $v(s, t)$ удовлетворяет предельному соотношению

$$
\lim _{s \rightarrow-\infty}(v(s, t)-f(s))=0
$$

где $f(s)$ - корень кубического уравнения

$$
f^{3}+f-s=0
$$

который при всех достаточно больших значениях $|s|$ разлагается в сходящийся ряд

$$
f(s)=s^{1 / 3}\left(1+\sum_{j=2}^{\infty} f_{j} s^{-j / 3}\right) .
$$

Сначала будет построено и обосновано равномерное по аргументу $s$ полное асимптотическое разложение при $t \rightarrow-\infty$ функции $v(s, t)$.

Формальное асимптотическое решение задачи (3.2), (3.3) легко находится в виде ряда

$$
v_{-}(s, t)=\sum_{k=0}^{\infty}|t|^{-5 k / 2} v_{k}(s),
$$

главный член которого

$$
v_{0}(s)=f(s)
$$

есть единственный корень кубического уравнения (3.4), а остальные выражаются через него из рекуррентной последовательности уравнений, возникающей после подстановки ряда (3.6) в ОДУ (3.2) и приравнивания в результате членов при одинаковых степенях $|t|$ :

$$
\begin{gathered}
v_{1}(s)\left(3 f^{2}(s)+1\right)=-v_{0}^{\prime}(s), \\
v_{k}(s)\left(3 f^{2}(s)+1\right)=-v_{k-1}^{\prime}(s)-\sum_{i_{1}+i_{2}+i_{3}=k, 0 \leqslant i_{j}<k} v_{i_{1}}(s) v_{i_{2}}(s) v_{i_{3}}(s), \quad k>0 .
\end{gathered}
$$

ЛЕмма 3.1. Функиии $v_{k}(s)$, определяемые соотношениями (3.7), (3.8), при всех достаточно больших значениях $|s|$ представляются сходящимися рядами

$$
v_{k}(s)=s^{(1-5 k) / 3} \sum_{j=0}^{\infty} c_{j k} s^{-j / 3} .
$$

ДокАЗАТЕЛЬСтво. Для функции $v_{0}(s)$ данный ряд есть ряд (3.5), а для остальных функций $v_{k}(s)$ справедливость разложений (3.9) легко обосновывается с помощью использования рекуррентных уравнений (3.8) и математической индукции. 
ЛЕмма 3.2. Для всех функиий $v_{k}(s)$ существуют такие положительные постоянные $C_{k}$, что при $s \in \mathbb{R}$ имеют место оценки

$$
\left|v_{k}(s)\right| \leqslant C_{k}\left(1+s^{2}\right)^{(1-5 k) / 6} .
$$

ДокАЗАТЕльство вытекает из справедливости разложения (3.9) при больших $|s|$ и из гладкости обеих частей неравенства (3.10).

Введем в рассмотрение функции

$$
\begin{aligned}
V_{n}^{-}(s, t) & =\sum_{k=0}^{n-1} \frac{v_{k}(s)}{|t|^{5 k / 2}}-\frac{K_{n}}{|t|^{5 n / 2}}\left(1+s^{2}\right)^{(1-5 n) / 6}, \\
V_{n}^{+}(s, t) & =\sum_{k=0}^{n-1} \frac{v_{k}(s)}{|t|^{5 k / 2}}+\frac{K_{n}}{|t|^{5 n / 2}}\left(1+s^{2}\right)^{(1-5 n) / 6} .
\end{aligned}
$$

Лемма 3.3. Функиии $V_{n}^{-}(s, t) u V_{n}^{+}(s, t)$ удовлетворяют условиям

$$
\lim _{s \rightarrow-\infty}\left(V_{n}^{-}(s, t)-f(s)\right)=\lim _{s \rightarrow-\infty}\left(V_{n}^{+}(s, t)-f(s)\right)=0 .
$$

Пусть $T>0$. При $n \in \mathbb{N}$ для достаточно больших значений постоянных $s_{n}, T_{n}, K_{n}$ в полуплоскости $t<-T_{n}$ и в областях

$$
|s| \geqslant s_{n}, \quad t<-T
$$

справедливы неравенства

$$
\begin{aligned}
& |t|^{-5 / 2}\left(V_{n}^{-}(s, t)\right)_{s}^{\prime}+\left(V_{n}^{-}(s, t)\right)^{3}+V_{n}^{-}(s, t)-s \leqslant 0, \\
& |t|^{-5 / 2}\left(V_{n}^{+}(s, t)\right)_{s}^{\prime}+\left(V_{n}^{+}(s, t)\right)^{3}+V_{n}^{+}(s, t)-s \geqslant 0 .
\end{aligned}
$$

ДокАзАтЕльство. Неравенства (3.13), (3.14) доказываются вполне аналогично тому, как в теореме 2.2 были доказаны неравенства (2.11), (2.12).

Если обозначить

$$
B_{n}(s, t)=\sum_{k=0}^{n-1} \frac{v_{k}(s)}{|t|^{5 k / 2}},
$$

то

$$
\begin{aligned}
& |t|^{-5 / 2}\left(V_{n}^{-}(s, t)\right)_{s}^{\prime}+\left(V_{n}^{-}(s, t)\right)^{3}+V_{n}^{-}(s, t)-s \\
& =|t|^{-5 / 2}\left(B_{n}(s, t)\right)_{s}^{\prime}+\left(B_{n}(s, t)\right)^{3}+B_{n}(s, t)-s \\
& \quad-\frac{K_{n}}{|t|^{5(n+1) / 2}} \frac{(1-5 n)}{6} 2 s\left(1+s^{2}\right)^{(1-5 n) / 6-1} \\
& \quad-3\left(B_{n}(s, t)\right)^{2} \frac{K_{n}}{|t|^{5 n / 2}}\left(1+s^{2}\right)^{(1-5 n) / 6}+3 B_{n}(s, t) \frac{K_{n}^{2}}{|t|^{5 n}}\left(1+s^{2}\right)^{(1-5 n) / 3} \\
& \quad-\frac{K_{n}^{3}}{|t|^{15 n / 2}}\left(1+s^{2}\right)^{(1-5 n) / 2}-\frac{K_{n}}{|t|^{5 n / 2}}\left(1+s^{2}\right)^{(1-5 n) / 6} .
\end{aligned}
$$


В силу рекуррентной последовательности соотношений (3.8) и оценок (3.10) функций $v_{k}(s)$ левая часть доказываемого неравенства (3.13) равна

$$
\begin{aligned}
& -\frac{K_{n}}{|t|^{5 n / 2}}\left(1+3 f^{2}(s)\right)\left(1+s^{2}\right)^{(1-5 n) / 6}+\sum_{j=n}^{3 n-3} \frac{y_{j}(s)}{|t|^{5 j / 2}}+K_{n} \sum_{j=n+1}^{3 n-2} \frac{y_{j, 1}(s)}{|t|^{5 j / 2}} \\
& \quad+K_{n}^{2} \sum_{j=2 n}^{3 n-1} \frac{y_{j, 2}(s)}{|t|^{5 j / 2}}-\frac{K_{n}^{3}}{|t|^{15 n / 2}}\left(1+s^{2}\right)^{(1-5 n) / 2},
\end{aligned}
$$

где

$$
\left|y_{j}(s)\right|+\left|y_{j, 1}(s)\right|+\left|y_{j, 2}(s)\right| \leqslant M_{j}\left(1+s^{2}\right)^{(1-5 j) / 6+1 / 3} .
$$

Выбирая сначала большое $K_{n}$, а затем достаточно большие $T_{n}$ (при рассмотрении полуплоскости $t<-T_{n}$ ) и $s_{n}$ (при рассмотрении областей $(3.12)$ ), получаем неравенство (3.13).

Доказательство неравенств (3.14) от приведенного доказательства неравенств (3.13) практически ничем не отличается.

Предельные же соотношения (3.11) являются очевидным следствием разложимости при $s \ll-1$ функций $v_{k}(s)$ в ряды (3.5), (3.9). Лемма доказана.

Теорема 3.1. Пусть $T>0$ - достаточно большое число. При $t \leqslant-T$ для любого $n \in \mathbb{N}$ найдется такая постоянная $C_{n}$, что справедливо неравенство

$$
\left.\left.\left|v(s, t)-\sum_{k=0}^{n-1}\right| t\right|^{-5 k / 2} v_{k}(s)\left|\leqslant C_{n}\right| t\right|^{-5 n / 2}\left(1+s^{2}\right)^{(1-5 n) / 6} .
$$

ДоказАтельство. Из вида уравнения (3.2) и из неравенства (3.14) следует, что для достаточно больших значений постоянных $s_{n}, T_{n}, K_{n}$ в полуплоскостях $t<-T_{n}$ и в областях (3.12) разность $R(s, t)=v(s, t)-V_{n}^{+}(s, t)$ удовлетворяет неравенству

$$
|t|^{-5 / 2} R_{s}^{\prime}(s, t)+R(s, t) Q(s, t)<0,
$$

где

$$
Q(s, t)=\left(V_{n}^{+}(s, t)\right)^{2}+V_{n}^{+}(s, t) v(s, t)+v^{2}(s, t)+1>1 .
$$

Кроме того, соотношения (3.3) и (3.11) означают, что выполнено условие

$$
\lim _{s \rightarrow-\infty} R(s, t)=0 .
$$

Поэтому использование леммы 1.2 позволяет сделать вывод о том, что при достаточно больших значениях $T_{n}$ и $s_{n}$ в полуплоскостях $t<-T_{n}$ и в левых частях областей (3.12), определяемых неравенствами $s \leqslant-s_{n}$, имеют место неравенства $v(s, t) \leqslant V_{n}^{+}(s, t)$. Точно так же показывается, что при этом справедливыми можно сделать и неравенства $v(s, t) \geqslant V_{n}^{-}(s, t)$.

Согласно теореме 2.2 на компактах

$$
|s| \leqslant s_{n}, \quad-T_{n} \leqslant t \leqslant-T
$$


модуль функции $v(s, t)$ ограничен некоторыми постоянными $G_{n}$. И так как на компактах (3.16) функции $B_{n}(s, t)$ и $|t|^{5 n / 2}\left(1+s^{2}\right)^{(1-5 n) / 6}$ гладкие, то утверждение о справедливости на этих компактах неравенств (3.15) тривиально.

Утверждение теоремы осталось доказать лишь для областей (3.12), определяемых неравенствами $s>s_{n}$ и $-T_{n}<t<T$. Это легко сделать, учитывая результаты лемм 1.3, 3.3 и ограниченность функций $v(s, t)$ и $V_{n}^{ \pm}(s, t)$ при $s=s_{n}$. Теорема доказана.

ТеОрема 3.2. Пусть $T>0$ - достаточно большое число. При $t \leqslant-T$ для любых натуральных $k, m, n$ найдутся такие постоянные $C_{k, m, n}$, что имеют место оченки

$$
\begin{aligned}
& \left|\frac{\partial^{k+m}}{\partial x^{k} \partial t^{m}}\left(u(x, t)-|t|^{1 / 2} \sum_{j=0}^{n-1}|t|^{-5 j / 2} v_{j}(s)\right)\right| \\
& \quad \leqslant C_{k, m, n}|t|^{(1-3 k-2 m-5 n) / 2}\left(1+s^{2}\right)^{(1-3 k-4 m-5 n) / 6} .
\end{aligned}
$$

ДокАЗАТЕЛЬСТво. При $k=m=0$ частным случаем доказываемой теоремы является теорема 3.1 .

Докажем справедливость доказываемой теоремы при $k=0$ и произвольных $m, n$. Для этого заметим, что при $m \geqslant 1$ производные

$$
q_{m}(x, t)=\frac{\partial^{m}}{\partial t^{m}} u(x, t)
$$

являются решениями рекуррентной последовательности линейных ОДУ

$$
\begin{aligned}
& \frac{\partial q_{m}}{\partial x}(x, t)=\left(t-3 u^{2}(x, t)\right) q_{m}(x, t)+m q_{m-1}(x, t) \\
& -\sum_{i_{1}+i_{2}+i_{3}=m, 0 \leqslant i_{j}<m} q_{i_{1}}(x, t) q_{i_{2}}(x, t) q_{i_{3}}(x, t), \quad m>0
\end{aligned}
$$

коэффициенты и правые части которых зависят только от параметра $t$ и функций $q_{j}(x, t)$ с индексами $j<m$.

После замен

$$
x=s|t|^{3 / 2}, \quad q_{m}(x, t)=|t|^{1 / 2-m} \rho_{m}(s, t), \quad u(x, t)=|t|^{1 / 2} \rho_{0}(x, t)
$$

уравнения (3.17) примут вид рекуррентной последовательности сингулярно возмущенных ОДУ

$$
\begin{array}{r}
|t|^{-5 / 2} \frac{\partial \rho_{m}}{\partial s}(s, t)=-\left(1+3 \rho_{0}^{2}(s, t)\right) \rho_{m}(s, t)+m \rho_{m-1}(s, t) \\
-\sum_{i_{1}+i_{2}+i_{3}=m, 0 \leqslant i_{j}<m} \rho_{i_{1}}(s, t) \rho_{i_{2}}(s, t) \rho_{i_{3}}(s, t), \quad m>0,
\end{array}
$$


решения которых согласно утверждению теоремы 2.5 удовлетворяют предельным соотношениям

$$
\lim _{s \rightarrow-\infty} \rho_{m}(s, t)=0 .
$$

Равномерная по переменной $s$ асимптотика функции $\rho_{0}(s, t)=v(s, t)$ при $t \rightarrow-\infty$ описана в теореме 3.1 - она имеет вид степенного ряда

$$
\rho_{0}(s, t)=\sum_{n=0}^{\infty} \rho_{0, n}(s)|t|^{-5 n / 2} .
$$

Из асимптотического разложения (3.20) по индукции находятся единственные формальные решения линейных ОДУ (3.18) вида

$$
\rho_{m}^{-}(s, t)=\sum_{n=0}^{\infty} \rho_{m, n}(s)|t|^{-5 n / 2} .
$$

Их коэффициенты задаются формулами

$$
\begin{aligned}
\rho_{m, n}(s, t)= & \frac{1}{1+3 f^{2}(s)}\left(-\left(\rho_{m, n-1}(s)\right)_{s}^{\prime}+m \rho_{m-1, n}(s)\right. \\
& \left.-\sum_{i_{1}+i_{2}+i_{3}=m}^{0 \leqslant i_{j}<m} \sum_{n_{1}+n_{2}+n_{3}=n}^{0 \leqslant n_{j} \leqslant n} \rho_{i_{1}, n_{1}}(s) \rho_{i_{2}, n_{2}}(s) \rho_{i_{3}, n_{3}}(s)\right) .
\end{aligned}
$$

Из свойств коэффициентов $\rho_{0, n}(s)=v_{n}(s)$, описанных в леммах 3.1 и 3.2, и последовательности формул (3.22) следует, что все функции $\rho_{m, n}(s)$ бесконечно дифференцируемы для любого $s$ и при достаточно больших значениях $|s|$ представляются сходящимися рядами

$$
\rho_{m, n}(s)=s^{(1-2 m-5 n) / 3}\left(a_{m, n}^{0}+\sum_{j=1}^{\infty} a_{m, n}^{j} s^{-j / 3}\right) .
$$

Стало быть, для них справедливы оценки

$$
\left|\frac{d^{k}}{d s^{k}} \rho_{m, n}(s)\right|=O\left(\left(1+s^{2}\right)^{(1-3 k-4 m-5 n) / 6}\right),
$$

гарантирующие, в частности, формальное удовлетворение рядом (3.21) предельному соотношению (3.19).

Из свойств (3.22)-(3.24) коэффициентов $\rho_{m, n}(s)$ формального решения задачи (3.18), (3.19) вытекает справедливость утверждения теоремы для $k=0$. Для проверки этого утверждения достаточно провести те же рассуждения, которые были использованы при доказательстве теоремы 3.1. Справедливость теоремы 3.2 для произвольных индексов $k$ доказывается вполне аналогично доказательству теоремы 2.5 . 


\section{Список литературы}

[1] А. М. Ильин, Б. И. Сулейманов, "О двух специальных функциях, связанных с особенностями типа сборки", Докл. РАН, 387:2 (2002), 156-158.

[2] В. И. Арнольд, Теория катастроф, Наука, М., 1980.

[3] А. М. Ильин, Б.И. Сулейманов, "Зарождение контрастных структур типа ступеньки, связанное с катастрофой сборки", Матем. сб., 195:12 (2004), 27-46.

[4] С. А. Чаплыгин, Новый метод приближенного интегрирования дифференииальных уравнений, Гостехиздат, М.-Л., 1950.

А. М. Ильин (А. М. Il'in)

Челябинский государственный университет

E-mail: iam@csu.ru, ilam@list.ru

Б. И. Сулейманов (В. I. Suleimanov)

Институт математики с ВЦ РАН, г. Уфа

E-mail: bis@imat.rb.ru, bisul@mail.ru 